\title{
Is Good More Alike as Bad? Positive-Negative Asymmetry in the Differentiation Between Options. Study on the Perception of Political Candidates
}

Magdalena Jabłońska ( $\sim$ mjablonska2@swps.edu.pl )

University of Social Sciences and Humanities

Andrzej Falkowski

University of Social Sciences and Humanities

Robert Mackiewicz

University of Social Sciences and Humanities

\section{Research Article}

Keywords: positive-negative asymmetry, similarity judgements, object differentiation, candidate evaluation

Posted Date: January 20th, 2022

DOI: https://doi.org/10.21203/rs.3.rs-1193574/v1

License: (c) (i) This work is licensed under a Creative Commons Attribution 4.0 International License. Read Full License 


\section{Abstract}

In our research, we investigate how well people differentiate between a few positive and negative options. Typically, negative entities are said to be more differentiated than their positive counterparts due to higher sensitivity potentially threatening stimuli. Contrary to that, we predict the opposite effect - a better differentiation of positive and not negative options - assuming that apart from being loss-avoidant people are also motivated to choose the best possible alternatives. Additionally, we investigate how positive and negative information affect object evaluation, predicting further asymmetries in object evaluations. In a series three experimental studies set in a political context, participants evaluated candidate profiles that differed in their overall valence. We used a numeric and narrative presentation of candidates as well designed evaluation tasks as either individual or joint assessments. Our findings provided strong evidence for a better differentiation between positive than negative options which was found in all three studies. It turns out that after exceeding a certain, relatively small level of negativity, people do not see any further increase in negativity. The increase in positivity, on the other hand, is more gradual, with greater differentiation among positive options. Our findings are discussed in light of such concepts as cognitive-experiential self-theory and density hypothesis.

\section{Introduction}

\subsection{Positive-negative asymmetry and negative differentiation}

People are constantly making evaluations of people they encounter and determine how good or bad they are ${ }^{1}$. However, the categories of "good" and "bad" are not symmetrical. Typically, people approach other

people and neutral objects in a positive manner ${ }^{2-4}$. Still, negative features have been repeatedly found to be more potent, intensive, dominant and informative ${ }^{5,6}$. The effect is known as positive-negative asymmetry or valence asymmetry.

Negative entities are also said to be more differentiated, with more complex conceptual representations and a wider response repertoire ${ }^{6}$. For instance, studies on word frequencies have shown that whereas positive words appear in written and spoken language more often ${ }^{7}$, the repertoire of words used to describe negative events and states is more diverse and varied ${ }^{8}$. The observation is addressed in density hypothesis, according to which a piece of positive information is more similar to another piece of positive information (and thus the set of positive information is denser), while the structure of negative information is more differentiated ${ }^{9}$.

A greater differentiation of negative entities is typically attributed to its higher adaptive value. Whereas positive stimuli generally produce approach reactions, an encounter with negative entities requires a more elaborate cognitive appraisal and may lead to diverse behavioural outcomes such as fight/ flight/ freeze responses ${ }^{6}$. Furthermore, studies of economic decision-making show that people react stronger to losses 
than gains ${ }^{10,11}$. For example, they accept higher levels of risk to avoid a loss than to get the gain of the same net value. Generally the ratio between the steepness for losses is 2.5 higher than the steepness for gains ${ }^{12}$ and has been also observed in non-economic contexts ${ }^{13,14}$.

\subsection{Research gap and study rationale}

Although the greater differentiation of negative entities is adaptive for reasons already discussed, it is possible to imagine situations in which the differentiation between positive objects is more beneficial. Apart from loss avoidance, people are also motivated by gain-seeking ${ }^{15}$. Thus after dismissing all unattractive options, people may be motivated to look for the best alternative from attractive options that are left. These predictions have been supported for example in consumer context in the research on the effect of additional positive and negative features ${ }^{16,17}$. It turned out that the participants deferred their choice more if products differed in negative but not positive options. Although these observations suggest that people see more difference between favourable options and less of a difference between unfavourable ones, this issue has not been addressed by the researchers in consumer setting. Similarly, the perception of difference among positive and negative options has not been an object of investigation in social context, despite numerous studies in the effect of particular favourable and unfavourable traits on person perception ${ }^{18-23}$. We identify this scarcity of research as an interesting research gap.

In our study we asked the participants to evaluate fictional political candidates running in parliamentary elections. We believe that although the differentiation between various people takes place in many social contexts (e.g. selection of potential romantic partners or employees during a job interview), the evaluation of political candidates is a process that most people at least occasionally undertake and which has important consequences on the functioning of states and the lives of others. Furthermore, political candidates are often presented in a form of comparison lists or rankings what fits well our research design.

The research on person perception often lacks precision found in economic decision-making. For example, it is relatively easy to compare changes in objective values such as variations in price with subjective evaluations of the magnitude of these changes such as estimates of how expensive something is. In social context, such objective measures are more difficult to find. Still, they are necessary if one wants to compare how well people differentiate between positive and negative options. Thus, in our research we propose a numeric and a narrative presentation of stimuli, both of which can be quantified. We also use similarity to an ideal and bad politician as measures of candidate evaluation. We believe that these two categories create natural comparison standards for candidates running in the elections.

\subsection{Hypotheses and research design:}

In three experiments, we test how well people differentiate between favourable and unfavourable candidate profiles. We predict that despite being hardwired to pick up on the bad stuff, people are also gain-driven and strive for best possible solutions. If all available alternatives are unappealing, it may not 
really matter which of them is worse. After all, all of them are equally bad (or at least seem to be). If, however, the assessment pertains to attractive options, then the appraisal of which of them is better gains on importance. Thus, that the following is predicted: :

\section{Hypothesis 1}

The differentiation between a good and better candidate will be greater than the differentiation between a bad and a worse candidate.

If so, we predict that a political candidate who has a high number of good features will be evaluated as better than the one with an average number of good features. Whereas, a candidate with an average number of bad features will be evaluated as badly as the one with a high number of bad features. The effect should hold for all measures used in the study (similarity to an ideal bad politician, affective evaluation and voting intention).

Furthermore, we want to analyse how some additional positive (or negative) pieces of information change the evaluation of a candidate whose image is favourable (i.e. has more positive traits than negative ones) and of that who is perceived unfavourably. Thus, we assume that the overall valence of an object (how favourable it is) will moderate the effect of additional (un)favourable features. We predict that some negative information will hurt the image of a good politician more than that of an already bad one. The effect can be explained with psychophysical laws such as the ratio difference principle $12,24,25$ which predicts that a less frequent feature will stand out and lead to greater changes in the evaluation. The principle is also incorporated into Tversky's contrast model of similarity ${ }^{26}$ which can be used to describe the affinity between two items based on the ratio of common and distinctive features. According to the feature-based model, a political candidate can be regarded as a set of positive and negative features that describe him or her. Depending on the favourability of candidate image (i.e. the ratio of positive and negative features), additional favourable or unfavourable pieces of information will lead to divergent changes in evaluations ${ }^{27,28}$. For instance, although positive features will make all candidates more attractive, the improvement will be more visible in the case of bad candidates than the already good ones. Thus, the following is predicted:

\section{Hypothesis 2}

Additional positive features will improve an image of a unfavourable politician more than an image of a favourable one, whereas additional negative features will degrade an image of a favourable politician more than an image of a unfavourable one.

The feature-based contrast model of similarity ${ }^{26}$ is normative and allows for precise calculations of changes in similarity between objects. The model, however, is valence-insensitive as it is based on the ratio between common and distinctive features and it does not account for positive-negative asymmetry. According to normative predictions, two positive features added to an object represented by two positive and four negative features should lead to comparable changes as those produced by two negative 
features added to an object characterized by two negative and four positive features. However, the empirical results tend to diverge from these normative predictions ${ }^{28}$. Thus, we predict that positive features will have a greater effect on the image of a favourable candidate than the negative features on the image of an unfavourable candidate.

\section{Hypothesis 3}

Additional negative features will not degrade an image of a unfavourable politician, whereas additional positive features will improve an image of a favourable politician

The predictions will be tested in three experimental studies. In Study 1 and 2 we will present the features of candidates as a set of dimensions (numeric presentation), whereas in Study 3 we will use narrative descriptions of candidates. Additionally, in Study 1 and 3 each candidate will be evaluated separately and later compared with others, whereas in Study 2 participants will be presented with pairs of candidate profiles and asked about the extent of difference between them.

\section{Study 1}

\section{Method:}

\subsection{Participants}

All studies were conducted in compliance with APA ethical guidelines ${ }^{29}$. The research protocols were approved by the Research Ethics Committee of the University of Social Sciences and Humanities, Warsaw, Poland (approval number 12/2020), an institution at which research was conducted. Informed consent was obtained from all participants. Forty two participants took part in the first experiment. The sample $(72.3 \%$ female) was relatively young $(M=25.10 ; S D=8.378)$ and was not remunerated for participation. On average, participants were slightly disinterested in politics (measured with a 11-point Likert scale, with 0 not at all interested in politics and 10 extremely interested in politics, $M=3.86, S D=$ 2.619) and were neither extremely left- or right-wing oriented (measured with a 11-point Likert scale, with 0 extreme left and 10 extreme right $M=4.55, S D=1.310$ ).

\subsection{Procedure}

The group was randomly divided into two research conditions depending on whether participants evaluated presented candidates with regard to their similarity to an ideal or bad politician. The division was introduced to account for potential framing effects, where the activation of certain categories (e.g. an ideal or bad object) serves as a natural reference point and may influence later evaluations of other objects ${ }^{15,30}$ At the beginning of the experiment, participants were instructed that they would be presented with profiles of five political candidates that ran in parliamentary elections. They were also informed that each candidate would be described with regard to six different dimensions (such as competence and honesty) and that each dimension could take the values of -10 to +10 , where the higher positive value 
symbolized the greater extent to which a politician possessed a certain positive feature of a particular dimension. Participants were asked to study each candidate profile individually and evaluate it. Each profile was presented separately and the order of the presentation was randomized. Participants could not return to their previous answers. After filling in demographic information, participants were debriefed.

\subsection{Materials}

The participants were presented with five candidate profiles that differed in the extent to which they possessed features relevant for a political post. Based on literature review, such aspects as education, qualifications, resourcefulness, honesty, justice and truthfulness were selected ${ }^{31-33}$. The characteristics were found to be the most common criteria in candidate evaluation. Each of the characteristics was presented on a 21 point bipolar scale, so that zero constituted the neural point, negative values pertained to the negativity of a feature and positive values to its positivity (e.g. for intelligence, -10 signified very low intelligence and +10 very high intelligence). Apart from a neutral candidate whose combined sum of measures on each of the scales equalled 0 , there were two negative and two positive candidate profiles. Positive candidate profiles were constructed in such a way that they had either 24 or 48 points towards the positive dimension, whereas negative profiles had either 24 or 48 points towards the negative dimension. We used the same descriptions in Experiment 2. In what follows we will use the following notation to refer to the candidates presented in Study 1 and 2:

Candidate +48 : the sum of scores on all dimensions equals minus 48

Candidate -24 : the sum of scores on all dimensions equals minus 24

Candidate 0 : the sum of scores on all dimensions equals 0

Candidate $+24:$ the sum of scores on all dimensions equals plus 24

Candidate $+48:$ the sum of scores on all dimensions equals plus 48

Table 1 illustrates the overall valence of candidate profiles used in Study 1. For participants, each candidate was presented individually in a tabular form.

Table 1: Candidate profiles used in Study 1. The numbers represent the extent to which a candidate possessed particular features on a scale -10 to +10 . 


\begin{tabular}{|llllll|}
\hline & Candidate -48 & Candidate -24 & $\begin{array}{l}\text { Candidate } \\
0\end{array}$ & Candidate +24 & Candidate +48 \\
\hline Resourcefulness & -6 & -2 & 2 & 2 & 6 \\
\hline Education & -10 & -6 & -2 & 6 & 10 \\
\hline Qualifications & -8 & -4 & 0 & 4 & 8 \\
\hline Honesty & -10 & -6 & -2 & 6 & 10 \\
\hline Justice & -7 & -3 & 1 & 3 & 7 \\
\hline Truthfulness & -7 & -3 & 1 & 3 & 7 \\
\hline
\end{tabular}

\subsection{Measures}

Participants evaluated each candidate individually with regard to their similarity to either an ideal or bad politician (depending on the experimental group). The question read: On a scale from 0 to 10 how similar is the candidate to an image of an ideal (bad) politician?, with answers ranging from 0 very dissimilar to 10 very similar.

\section{Results and Discussion}

Figure 1 presents mean ratings for candidates' similarity to an ideal and bad politician.

The aim of Study 1 was to test Hypothesis 1 and 3. The initial analysis showed that for most of the variables the data were not normally distributed and exceeded the accepted levels of skewness ${ }^{34}$. Thus, instead of a repeated measures analysis of variance, its non-parametric version, Friedman's test was used 35 in order to test whether candidate valence $(-48,-24,0+24,+48)$ differentiated profiles with regard to their similarity to an ideal and bad politician. The results of the analysis were significant for both types of similarity, showing significant differences between candidate profiles with regard to their similarity to an ideal politician, $\chi^{2}(4)=71.543, p<0.001$, and similarity to a bad politician, $\chi^{2}(4)=59.276, p<0.001$.

As the data contained many similar ratings (e.g. zeros as similarity of the worst candidate compared to the ideal politician) instead of traditional parametric or non-parametric testing, permutation tests were used for the post-hoc checks ${ }^{36,37}$. The probabilities and confidence intervals for re-sampled groups were obtained with the resample package available for R Studio ${ }^{38}$. Additionally, we used Cohen's $d$ as a measure of the effect size ${ }^{39}$.

The results for similarity to an ideal politician showed significant differences between candidates -24 and $0(p<0.001, d=-0.895, C l=-1.792-0.002), 0$ and $+24(p<0.001, d=2.094, C l=-1.03-3.158)$ as well as +24 and $+48(p<.001, d=-0.97, C l=-1.875--0.066)$. There was no difference between candidates -48 and $-24(p=.447, d=0.188, C l=-0.669-1.045)$. For similarity to a bad politician, there were significant 
differences between candidates 0 and $-24(p<0.001, d=-0.768, C l=-1.655-0.118)$ as well as 0 and +24 $(p<0.001, d=-1.9, C l=-0.869-2.93)$. No differences between candidates -48 and $-24(p=.483, d=0.164$, $C l=-0.693-1.021)$ as well as +24 and $+48(p=.171, d=-0.309, C l=-1.169-0.552)$ were found. We show all significant differences in Figure 1. The analysis of effect sizes showed greater differences between favourable candidate profiles (i.e. candidates +24 and +48 , with Cohen's $d$ equal 0.97 for similarity to an ideal politician and 0.309 for similarity to a bad politician) than unfavourable candidates (i.e. candidates -24 and -48 , with $d=0.188$ and $d=.164$ respectively), providing evidence for Hypothesis 1 . Furthermore, corroborating the predictions of Hypothesis 3 , the study showed that an increase in feature negativity did not deteriorate candidate evaluation for candidates who were already perceived as unattractive, whereas an increase in feature positivity improved the evaluation of already favourable candidates. The effect, however, was limited to the similarity to an ideal politician as a dependent variable and was not present in the similarity to a bad politician.

\section{Study 2}

In Study 2, we wanted to test whether the differences between candidate profiles are restricted only to similarity measures or whether they are also observed in other measures important for candidate evaluation. Thus, we added affective evaluation and voting intention as two additional dependent variables. Furthermore, although the results of Study 1 showed greater differences between favourable candidate profiles, participants evaluated each candidate individually, so that there was no direct comparison. Thus, in Study 2 we presented two candidate profiles (either a favourable candidate and its improved version or an unfavourable one and its deteriorated version) one after another and asked participants to evaluate the perceived difference between them. Again, Hypothesis 1 and 3 were tested.

\section{Method:}

\subsection{Participants}

Forty three participants from the same population as those in Study $1\left(86 \%\right.$ female; $M_{\text {age }}=32.72 ; S D=$ $10.859)$ took part in the experiment. On average, participants were slightly disinterested in politics (measured as previously, $M=3.74, S D=2.656)$ and were neither extremely left- or right-wing oriented $(M=$ $4.63, S D=1.574)$.

\subsection{Procedure}

The procedure was similar to that described in Study 1 . This time, however, participants were divided into four research groups. First, as previously participants were divided into groups which evaluated either the similarity to an ideal or bad politician to account for framing effects. Later, each group was further divided into two groups, depending on candidate valence. Two groups were presented with favourable candidate profiles $(+24,+48$; the same as in Study 1$)$, whereas two others with unfavourable ones $(-24$, -48). Additionally, each group evaluated a neutral candidate whose score equalled 0 . Thus, each participant was presented with three candidate profiles. After reading one profile, participants evaluated it 
and moved to the other one. Each profile was presented separately and the order of the presentation was randomized. In the second part of the experiment, participants were presented with pairs of candidate profiles and asked to evaluate how similar they were.

\subsection{Materials}

The same candidate profiles as in Study 1 were used. In the first part of the experiment each candidate profile was presented individually. In the second part, the candidates were organized in six pairs. In the study, only the following pairs were compared: 0 and $+24,+24$ and $+48,0$ and -24 as well as -24 and -48 .

\subsection{Measures}

Similarity measures used in the first part of the experiment were the same as in Study 1. Additionally, participants evaluated each profile with regard to affective evaluation (measured with a question How much do you like the politician?, with answers ranging from 0 I dislike the politician a lot to 10 I like the politician a lot) and voting intention (If the politician ran for an office, how likely are you to vote for him?, with answers ranging from 0 / would definitely not vote for the politician to 10 / would definitely vote for the politician). The differentiation between options was measured with one question (On a scale from 0 to 10 how similar are the two candidates?) with answers ranging from 0 very dissimilar to 10 very similar.

\section{Results and Discussion}

In order to test Hypothesis 1 and 3, a mixed ANOVA was conducted, with candidate profile (0 vs 24 vs 48) as a within factor and candidate valence (positive $v s$ negative) and valence of the reference point (ideal $v s$ bad politician) as between factors. The means for similarity measures, affective evaluation and voting intention are presented in Table 2. In the table, the candidates are organized in pairs in which they were compared. A three-way interaction was significant for similarity measure, $F(2,38)=24.091, p<.001$, eta2 $=0.559$ and non-significant for affective evaluation and voting intention. For these two variables, significant candidate profile $\times$ candidate valence interaction effects were found, $F(2,38)=53,335, p<.001$, eta2 $=0.737$ and $F(2,38)=41.742, p<.001$, eta $2=0.687$. As expected post-hoc analyses showed no differences between candidates -24 and -48 and candidates 0 and -24 . But the tests revealed significant differences between candidates 0 and +24 and +24 and +48 . Table 2 presents the means for all candidate pairs analysed in the study along with the significance of differences between compared profiles.

Table 2: Means for candidate profiles analysed in Study 2. Candidate profiles are organized in pairs in which they were compared. 


\begin{tabular}{|c|c|c|c|c|c|c|c|}
\hline DV & $\begin{array}{l}\text { Reference } \\
\text { point }\end{array}$ & $\begin{array}{l}\text { Candidate pair } \\
\text { (1) } v s(2)\end{array}$ & $\begin{array}{l}M \\
(1)\end{array}$ & $\begin{array}{l}\text { SD } \\
(1)\end{array}$ & $\begin{array}{l}M \\
(2)\end{array}$ & $\begin{array}{l}\text { SD } \\
(2)\end{array}$ & Significance \\
\hline \multirow[t]{8}{*}{ Similarity } & \multirow{4}{*}{$\begin{array}{l}\text { An ideal } \\
\text { politician }\end{array}$} & $-48 v s-24$ & 1.67 & 3.025 & 1.25 & 1.913 & ns \\
\hline & & -24 vs 0 & 1.25 & 1.913 & 2.00 & 2.412 & ns \\
\hline & & $0 v s+24$ & 0.83 & 1.467 & 3.08 & 1.975 & ** \\
\hline & & $+24 v s+48$ & 3.08 & 1.975 & 6.75 & 2.340 & ** \\
\hline & \multirow{4}{*}{$\begin{array}{l}\text { A bad } \\
\text { politician }\end{array}$} & $-48 v s-24$ & 9.20 & 1.619 & 7.20 & 3.259 & ** \\
\hline & & -24 vs 0 & 7.20 & 3.259 & 6.30 & 3.093 & ns \\
\hline & & $0 v s+24$ & 7.56 & 2.603 & 4.22 & 2.108 & ** \\
\hline & & $+24 v s+48$ & 4.22 & 2.108 & 2.11 & 1.691 & ** \\
\hline \multirow[t]{8}{*}{$\begin{array}{l}\text { Affective } \\
\text { evaluation }\end{array}$} & $\begin{array}{l}\text { An ideal } \\
\text { politician }\end{array}$ & $-48 v s-24$ & 0.92 & 2.021 & 1.25 & 1.712 & ns \\
\hline & & -24 vs 0 & 1.25 & 1.712 & 2.00 & 2.216 & ns \\
\hline & & $0 v s+24$ & 1.42 & 1.505 & 3.92 & 2.193 & ** \\
\hline & & $+24 v s+48$ & 3.92 & 2.193 & 6.58 & 2.314 & ** \\
\hline & $\begin{array}{l}\text { A bad } \\
\text { politician }\end{array}$ & $-48 v s-24$ & 0.80 & 1.874 & 1.10 & 2.025 & ns \\
\hline & & -24 vs 0 & 1.10 & 2.025 & 2.40 & 2.221 & ns \\
\hline & & $0 v s+24$ & 1.78 & 1.093 & 4.22 & 2.279 & $\star \star$ \\
\hline & & $+24 v s+48$ & 4.22 & 2.279 & 7.56 & 1.236 & ** \\
\hline \multirow{8}{*}{$\begin{array}{l}\text { Voting } \\
\text { intention }\end{array}$} & \multirow{4}{*}{$\begin{array}{l}\text { An ideal } \\
\text { politician }\end{array}$} & $-48 v s-24$ & 0.92 & 2.109 & 1.50 & 2.067 & ns \\
\hline & & -24 vs 0 & 1.50 & 2.067 & 2.25 & 2.491 & ns \\
\hline & & $0 v s+24$ & 1.00 & 1.595 & 3.42 & 2.575 & $\star \star$ \\
\hline & & $+24 v s+48$ & 3.42 & 2.575 & 6.75 & 2.491 & $\star \star$ \\
\hline & \multirow{4}{*}{$\begin{array}{l}\text { A bad } \\
\text { politician }\end{array}$} & $-48 v s-24$ & 1.40 & 2.547 & 0.80 & 1.317 & ns \\
\hline & & -24 vs 0 & 0.80 & 1.317 & 2.50 & 2.759 & ns \\
\hline & & $0 v s+24$ & 1.44 & 1.509 & 4.22 & 2.863 & $\star \star$ \\
\hline & & $+24 v s+48$ & 4.22 & 2.863 & 7.89 & 1.364 & ** \\
\hline
\end{tabular}

Note: ${ }^{*} p<0.01$ 
The study provided evidence for Hypothesis 3 , showing that whereas increase in feature negativity did not degrade the image of unfavourable candidates, increase in candidate positivity improved the evaluation of already favourable candidate profiles. Thus, the findings indirectly supported Hypothesis 1 , showing a better differentiation between favourable candidates than the unfavourable ones.

Furthermore, in order to directly test whether people see greater differences between positive than negative options, we analysed the responses for the perceived similarity between two candidates $(+24$ and +48 or -24 and -48 ) in direct comparisons, where participants evaluated on a 10-point Likert scale how similar the two candidates were. The descriptive statistics for the perceived similarity between the candidates are presented in Table 3 . In the table, the candidates are divided into four separate groups based on the valence of the activated category (an ideal/ bad politician) as well as the valence of the compared candidates (favourable/ unfavourable).

Table 3: The similarity between two candidates, favourable - $(+24$ and +48$)$ and unfavourable $(-24$ and -48) ones - measured on a 10-point Likert scale. Due to study manipulation used in the first part of the experiment, the statistics are presented separately for two research conditions (the activation of an ideal or bad politician as a reference point).

\begin{tabular}{|lllll|}
\hline Similarity between candidates & $N$ & $M$ & SD & SEE \\
\hline Positive reference point (ideal politician) & & & & \\
\hline similarity between $+24 v s+48$ & 12 & 6.25 & 2.598 & 0.750 \\
\hline similarity between $-24 v s-48$ & 12 & 4.17 & 2.758 & 0.796 \\
\hline Negative reference point (bad politician) & & & & \\
\hline similarity between $+24 v s+48$ & 10 & 6.40 & 2.951 & 0.933 \\
\hline similarity between $-24 v s-48$ & 9 & 6.22 & 2.489 & 0.830 \\
\hline
\end{tabular}

The results of $t$ tests showed that in the group in which a positive category was activated (an image of an ideal politician), the perception of difference between two good candidates ( +24 and +48$)$ was greater than the difference between two bad candidates (-24 and 48). The effect was marginally significant, $t(22)=1.905, p=.07$. When, however, participants first answered the question on candidates' similarity to a bad politician, the level of similarity between candidates +24 and +48 equalled the similarity between candidates -24 and -48 . Although such discrepancy between two experimental groups was unexpected, the finding corroborated the results of Study 1 , where almost no difference between candidates was found for similarity to a bad politician as a dependent variable. If so, the result indicates that it may be more difficult to spot differences between options (whether positive or negative) when the negative category is activated.

Overall, the results of Study 2 corroborated previous findings by showing that people see more difference between positive political candidates than the negative ones. As Study 2 showed, the effect holds not 
only for similarity measures but also affective evaluation and voting intention. Furthermore, the effect holds not only for individual assessments (Study 1) but also joint assessments (Study 2).

\section{Study 3}

Study 1 and Study 2 tested the differences in the perception of favourable and unfavourable candidates whose features were presented as a set of dimensions. In Study 3, we wanted to test whether the same effect would hold if candidates were described in a narrative form. So we designed narrative descriptions of eight candidate profiles that differed in the number of positive and negative features that characterized them. We divided the candidates into more favourable and unfavourable ones and organized them in pairs, so that one of the candidates had always either two positive or negative features more than the other one. By doing that, we were able to test how the same positive or negative features added to the description of differently valenced candidates influenced their perception. Thus, we were able to directly test not only Hypothesis 1 and 3, but also Hypothesis 2 .

\section{Method}

\subsection{Participants}

One hundred twenty participants, aged $18-54(M=24.41, S D=5.449)$ took part in the experiment. The sample (57\% female) was moderately interested in politics $(M=4.33, S D=2.696$, on a 11-point scale) and was neither extremely left- or right-wing oriented $(M=4.42, S D=1.943$, on a 11-point Likert scale, as measured on the same scale as in the two previous studies).

\subsection{Procedure}

Each participant was asked to read and evaluate the descriptions of two political candidates. Each candidate was evaluated individually and the order of the presentation was randomized.

\subsection{Materials}

Eight descriptions of political candidates were used. Each participant evaluated two candidates - one positive and one negative. The questionnaires were designed in such a way that both evaluated candidates had the same proportion of features but they differed in the extent of their positivity/ negativity. For instance, a participant would evaluate a candidate profile characterized by seven positive and two negative features and the other profile characterized by two positive features and seven negative features. The order of presentation was randomized. In total, eight different versions of questionnaires were prepared. One hundred twenty participants provided in total 240 evaluations of candidate profiles which were treated as independent measures due to randomization.

The candidates differed in the proportion of positive and negative features that described them. The number of features characterizing each candidate is presented in Table 4. The candidates are ordered from the most to the least favourable. 
Table 4: Candidate profiles used in Study 3 organized from the most to the least favourable. Image favourability is calculated as follows: the number of positive features divided by the number of all features characterizing a candidate.

\begin{tabular}{|llll|}
\hline Candidate & Positive features & Negative features & Image favourability \\
\hline A & 9 & 2 & 0.82 \\
\hline B & 7 & 2 & 0.78 \\
\hline C & 9 & 4 & 0.69 \\
D & 7 & 4 & 0.64 \\
\hline E & 4 & 7 & 0.36 \\
\hline F & 4 & 9 & 0.31 \\
\hline G & 2 & 7 & 0.22 \\
\hline H & 2 & 9 & 0.18 \\
\hline
\end{tabular}

The features were selected based on prior research in which we generated features characteristic for the category of an ideal and bad politician. We made sure that each favourable candidate had the same seven positive (cares for citizens, ensuring security, competent, good public speaker, stable in beliefs, consistent, ambitious) and two negative features (disloyal, greedy). Such a profile was treated as the base profile and it could differ from other favourable candidate profiles by either additional two positive (well-educated, committed) or negative features (lacking culture, not keeping election promises). The same rule applied to the unfavourable candidate profile, with seven negative (quarrelsome, lazy, greedy, populist, despotic, nepotistic, disloyal) and two positive (cares for citizens, ensures country security) features. If a candidate possessed two additional positive or negative features, they were the same as for favourable candidates. Additionally, in a pilot study we made sure that the image of favourable and unfavourable candidate (i.e. 7+2- vs 2+7-) did not differ when measured in absolute values when it comes to its image favourability (on a scale -10 to +10 ), $F(1,120)=3.105, p=0.098, \eta^{2}=0.172$. Similarly, there were no differences between additional two positive and negative features, $F(1,120)=2.04, p=0.173, \eta^{2}$ $=0.120$

\subsection{Measures}

Each participant evaluated a candidate in relation to five dependent variables. Similarity to an ideal and bad politician, affective evaluation and voting intention were measured as in Study 2 . Additionally, participants were asked to rate candidate image favourability (On a scale from -10 to +10 how would you rate candidate image favourability?, with -10 extremely unfavourable to +10 extremely favourable). 


\section{Results and Discussion}

Table 5 presents the means for dependent measures tested in Study 3.

Table 5: The means for affective evaluation, similarity measures and voting intention of candidates analysed in the study. Column 'candidate profile' summarizes the number of positive features (+) and negative features (-) used in their description.

\begin{tabular}{|lllllllllll|}
\hline $\begin{array}{l}\text { Candidate } \\
\text { profile }\end{array}$ & $\begin{array}{l}\text { Affective } \\
\text { evaluation }\end{array}$ & \multicolumn{2}{l}{$\begin{array}{l}\text { Similarity to } \\
\text { an ideal } \\
\text { politician }\end{array}$} & $\begin{array}{l}\text { Similarity to } \\
\text { a bad } \\
\text { politician }\end{array}$ & $\begin{array}{l}\text { Voting } \\
\text { intention }\end{array}$ & \multicolumn{2}{l|}{$\begin{array}{l}\text { Image } \\
\text { favourability }\end{array}$} \\
\hline $9+2-$ & $M$ & $S D$ & $M$ & $S D$ & $M$ & $S D$ & $M$ & $S D$ & $M$ & $S D$ \\
\hline $7+2-$ & 6.2 & 1.584 & 5.73 & 2.016 & 3.53 & 1.943 & 6.13 & 2.224 & 3.70 & 4.061 \\
\hline $9+4-$ & 5.72 & 2.153 & 5.55 & 2.063 & 4.03 & 2.163 & 5.86 & 2.356 & 3.86 & 3.378 \\
\hline $7+4-$ & 4.63 & 2.341 & 4.2 & 2.483 & 4.97 & 2.498 & 4.07 & 2.728 & 1.17 & 5.299 \\
\hline $4+7-$ & 4.48 & 2.204 & 3.90 & 2.119 & 5.00 & 2.236 & 3.94 & 2.065 & 0.67 & 4.566 \\
\hline $4+9-$ & 3.23 & 2.432 & 3.06 & 2.658 & 6.06 & 2.516 & 3.06 & 2.620 & -1.61 & 5.142 \\
\hline $2+7-$ & 2.87 & 1.978 & 2.17 & 1.733 & 6.93 & 2.273 & 2.30 & 1.968 & -2.97 & 4.586 \\
\hline $2+9-$ & 2.55 & 1.901 & 2.17 & 1.583 & 6.62 & 2.441 & 2.10 & 1.896 & -3.76 & 3.961 \\
\hline & 2.47 & 1.961 & 2.03 & 2.042 & 7.53 & 2.255 & 1.83 & 1.967 & -4.70 & 4.779 \\
\hline
\end{tabular}

The results of one-way ANOVA conducted separately for favourable and unfavourable candidates showed that the evaluation of favourable candidates was more differentiated than that of unfavourable ones. The effect was visible for all dependent variables, showing significant differences between favourable candidates in affective evaluation, $\mathrm{F}(3,116)=4.817, p=.002, \eta^{2}=0.111$, similarity to an ideal politician, $\mathrm{F}(3,116)=5.486, p=.001, \eta^{2}=0.124$, similarity to a bad politician, $\mathrm{F}(3,116)=3.194, p=.026$, $\eta^{2}=0.076$, voting intention $\mathrm{F}(3,116)=7.299, p<.001, \eta^{2}=0.159$, and image favourability $\mathrm{F}(3,116)=$ $4.275, p=.007, \eta^{2}=0.101$. However, no differences between unfavourable candidate profiles were found in any of the dependent variables, for affective evaluation, $\mathrm{F}(3,116)=.833, p=.479, \eta^{2}=0.021$, similarity to an ideal politician, $\mathrm{F}(3,116)=1.622, p=.188, \eta^{2}=0.041$, similarity to a bad politician, $\mathrm{F}(3,116)=$ 2.030, $p=.114, \eta^{2}=0.050$, voting intention $\mathrm{F}(3,116)=1.867, p=.139, \eta^{2}=0.046$, and image favourability $\mathrm{F}(3,116)=2.408, p=.071, \eta^{2}=0.059$. Thus, the results provided evidence for Hypothesis 1 .

Furthermore, in order to test which of the features - positive or negative - have a greater effect on candidate perception, we organized candidate profiles in pairs, so that the other candidate from the pair 
had two additional positive or negative features more than the first candidate. The first column in Table 6 presents how candidates were organized in pairs. In order to test the predicted effects, we conducted a series of $2 \times 4$ between-subject ANOVAs, separately for an increase in positive and negative features. The number of features was the first factor and the compared pair (two pairs for unfavourable profiles and two pairs for unfavourable ones; described below as candidate favourability) was the second factor. The results for all main and interaction effects are presented in Appendix 1 . Here we will focus on the results of planned comparisons that tested the predictions of Hypothesis 2 and Hypothesis 3. In Table 6 we present effect sizes for the effect of additional positive and negative features for selected candidate pairs.

Table 6: Effect sizes for the effect of additional positive and negative information items for candidate pairs analysed in Study 3. Significant differences in evaluations are marked by an asterisk.

\begin{tabular}{|c|c|c|c|c|c|c|}
\hline & & $\begin{array}{l}\text { Affective } \\
\text { evaluation }\end{array}$ & $\begin{array}{l}\text { Similarity to } \\
\text { the ideal }\end{array}$ & $\begin{array}{l}\text { Similarity to } \\
\text { the bad }\end{array}$ & $\begin{array}{l}\text { Voting } \\
\text { Intention }\end{array}$ & $\begin{array}{l}\text { Image } \\
\text { favorability }\end{array}$ \\
\hline $\begin{array}{l}\text { Additional } \\
\text { features }\end{array}$ & Pair & $d$ & $d$ & $d$ & $d$ & $d$ \\
\hline \multirow[t]{4}{*}{ Positive } & $\begin{array}{l}2+9-v s \\
4+9-\end{array}$ & 0.203 & 0.074 & -0.265 & 0.239 & 0.369 \\
\hline & $\begin{array}{l}2+7-v s \\
4+7-\end{array}$ & 0.31 & 0.404 & -0.226 & 0.418 & 0.466 \\
\hline & $\begin{array}{l}7+4-v s \\
9+4-\end{array}$ & 0.066 & 0.13 & -0.013 & 0.054 & 0.101 \\
\hline & $\begin{array}{l}7+2-\text { vs } \\
9+2-\end{array}$ & 0.255 & 0.088 & -0.243 & 0.118 & -0.043 \\
\hline \multirow[t]{4}{*}{ Negative } & $\begin{array}{l}2+7-v s \\
2+9-\end{array}$ & -0.041 & -0.076 & 0.388 & -0.14 & -0.214 \\
\hline & $\begin{array}{l}4+7-\text { vs } \\
4+9-\end{array}$ & -0.162 & -0.394 & 0.362 & -0.326 & -0.279 \\
\hline & $\begin{array}{l}7+2-\text { vs } \\
7+4-\end{array}$ & $-0.569 *$ & $-0.789 *$ & 0.441 & $-0.866^{\star}$ & $-0.796^{*}$ \\
\hline & $\begin{array}{l}9+2-v s \\
9+4-\end{array}$ & $0.788^{*}$ & $-0.678^{*}$ & $0.645^{\star}$ & $-0.829 *$ & $-0.537 *$ \\
\hline
\end{tabular}

Note: * marks confidence intervals significant at $95 \%$ level.

The results show that whereas additional positive features did not change candidate evaluation in any of the pairs, additional negative features decreased the evaluation of favourable candidates (i.e. candidates who had seven or nine positive features and two or four negative features) as can be visible in significant and large effect sizes. The only exception was the effect of additional negative features on similarity to a bad politician, where no difference between candidates 7+2- and 7+4- was found. The lack of the effect 
seems to be, however, an exception to a general rule and fit well the results of Study 1 and 2 where typically no differences between candidate profiles were found for similarity to a bad politician as a dependent variable. Thus, the results generally provided evidence for Hypothesis 2 (although the effect was restricted to positive and not negative features) but refuted the predictions of Hypothesis 3 , which anticipated that additional positive features would increase evaluation of already favourable candidates.

Overall, Study 3 again provided evidence for a better differentiation between favourable candidate profiles than the unfavourable ones. Additionally, the findings corroborated the negativity effect, showing negative features to be stronger than their positive counterparts. However, as predicted, the effect was limited to favourable candidate profiles. No effect of positive features was found, showing that additional positive information did not affect candidate evaluation, regardless of candidate image favourability. On the one hand, the finding is in line with ratio difference principle and contrast effects but on the other runs against the results of Study 1 and 2. This discrepancy between studies may be attributed to differences in candidate presentation. Perhaps, additional two positive adjectives carried less diagnostic information than their negative counterparts. If so, the effect follows the predictions of density hypothesis but it seems to be limited to linguistic attributes and not information presented in a numeric manner.

\section{General Discussion}

In a series of three experimental studies, we tested how well people differentiate among favourable and unfavourable options. We focused on the perception of difference between various more or less favourable fictitious political candidates. The political setting was chosen for two reasons. First, there is scarcity of research on the differentiation among positive and negative options in social context. Second, we believe that the evaluation of political candidates is a process that most people at least occasionally undertake and which has important social and political consequences.

Most research on negative differentiation predicts greater differences between negative entities than the positives ones due to a higher sensitivity to potentially threatening stimuli. Contrary to these assumptions, we anticipated an opposite effect, assuming that apart from loss avoidance people are also gain-driven and want to choose the best available options. If so, they will be less motivated to find out which of the options is the worst one than to decide which of the alternatives is the best one. Based on this rationale, we predicted greater differentiation among a few positive options than a few negative ones (Hypothesis 1). However, the effect that additional positive and negative information has on object evaluation is strictly connected to the valence of an object. According to psychophysical laws such as ratio-difference principle and the assumptions of the contrast model of similarity, a less frequent feature stands out and has a stronger effect than a more common feature. Thus, we predicted that additional positive features would improve the evaluation of a generally unfavourable object to a greater extent than the evaluation of a favourable object. The reversed was predicted for the effect of additional negative features (Hypothesis 2). Finally, we formulated a hypothesis that joined together the normative predictions assumed in Hypothesis 2 with a greater differentiation among positive options put forward in Hypothesis 1. Thus, in Hypothesis 3 we predicted that additional positive features would have a stronger 
effect on the evaluation of a favourable option than the negative ones on the evaluation of an favourable one.

In order to test these hypotheses, we conducted three studies. In Study 1 and 2 participants evaluated candidate profiles presented in a numeric manner. Each candidate was described in relation to six dimensions presented on a 21-point bipolar scale (with -10 as the maximal negative value and +10 as the maximal positive value). In total, there were five candidate profiles that differed in their valence. In both studies, participants evaluated candidates with regard to their similarity to an ideal and bad politician. Additionally, in Study 2 they assessed affective evaluation, voting intention and image favourability. In Study 1 participants appraised each candidate individually, whereas in Study 2 they were also asked to compare two candidates and rate the perceived similarity between them. In Study 3 candidates were presented in a narrative manner and participants read descriptions of fictitious political candidates that differed in the number of positive and negative features that described them. In total, there were eight candidates. The candidates were organized in pairs so that one of the candidates had always two additional positive (or negative) features more than the other one.

Generally, the results supported our hypotheses. In Study 1 and 2 we found that people saw no difference between negative candidates such as a candidate with overall score -24 and a candidate with overall score -48 (the numbers refer to the balance of the evaluations on five different dimensions). Still, the participants perceived candidates with overall scores +24 and +48 as significantly different. The effect was replicated in Study 3, in which candidates were described in a narrative form. Here, no differences were found for candidates whose descriptions mainly consisted of negative features. As expected, there were, however, significant differences between candidates whose profiles were generally positive. Thus, the findings provided a general support for Hypothesis 1 and 3. In all studies, the effect was present for the majority of tested measures such as affective evaluation, voting intention, similarity to an ideal and bad politician as well as image favourability. Moreover, we observed the same pattern of results in different experimental settings (separate $v s$ joint assessments as well as numeric $v s$ narrative presentation of objects), providing evidence for the greater perceived differences among positive options than their negative counterparts.

Our second research interest was to test the effect of additional positive and negative features on candidate evaluation depending on the favourability of candidate image. In light of the ratio difference principle and the contrast model of similarity, we expected additional positive features to have a more profound effect (compared to negative features) on the image on an unfavourable candidate. For favourable candidate profiles, negative features were hypothesized to be stronger than the positive ones. Accounting for the anticipated greater differentiation between positive options, we also predicted that positive features would have a greater effect on the image of a favourable candidate than the negative features on the image of an unfavourable candidate.

As expected, positive features increased candidate evaluation, whereas negative ones decreased it but these effects were not symmetrical, undermining the predictions of the contrast model of similarity. More 
precisely, Study 1 and Study 2 showed that a further increase in feature negativity did not deteriorate the evaluation of already unfavourable candidates, whereas an increase in feature positivity led to a noticeable improvement in the evaluation of favourable candidates. The results are important for two reasons. First, they present a limitation to the negativity effect, showing that after a certain amount of negativity any further increase produces no meaningful changes in object evaluation. Second, the findings are in line with previously mentioned asymmetry in the differentiation between positive and negative options.

The effect of additional positive and negative features on candidate evaluation was further analysed in Study 3. Here, negative features turned out to be stronger compared to their positive counterparts. Importantly, the effect was significant only for favourable candidate profiles. On the one hand, the findings can be interpreted with regard to the negativity effect. However, as we made sure to choose positive and negative features that were comparable in their valence intensity, we believe that the findings once again point to a better differentiation between positive options. In other words, although objectively identical in their intensity (as indicated in pre-tests), negative features turned out to be stronger, only when added to the characteristics of good candidates. Alternatively, no effect of additional positive features found in Study 3 may be also attributed to a different manner of object presentation. Namely, it is possible that two additional positive characteristics carried less information (i.e. were less diagnostic) than their negative counterparts. If so, the finding is in line with density hypothesis which predicts that a piece of positive information is more similar to another piece of positive information, while the structure of negative information is more differentiated. However, the research verifying density hypothesis was limited to studies on the internal differences between positive and negative linguistic forms and was not tested for the differentiation between objects.

Overall, our findings suggest that people do not see much of a difference between political candidates with many negative features, regardless of the extent to which they are presented as bad. The effect may be attributed to different motivations in the processing of positive and negative options. If all available alternatives are unappealing, it does not really matter which one of them is worse. However, if the judgment pertains to appealing options, then the decision which one of them is better gains on importance. The effect may be explained with regard to two independent information processing systems proposed by Epstein in his cognitive-experiential self-theory ${ }^{40,41}$. The evolutionally older experiential system operates in an automatic and holistic manner, whereas the rational system is "a relative newcomer on the evolutionary scene, is a deliberative, verbally mediated, primarily conscious analytical system that functions by a person's understanding of conventionally established rules of logic and evidence" (Denes-Raj \& Epstein, 1994, p. 819). Using this distinction, it seems that whereas an intense dislike towards negative options is an outcome of the experiential system, a better and more discriminative analysis of positive options is governed by the rational system.

This effect is also visible in very extreme evaluations of unfavourable candidates and more moderate and differentiated evaluations of favourable ones. As shown in Study 1, whereas it was relatively easy for bad candidates to be evaluated almost at the bottom of the scale, none of the good candidates seemed 
good enough to get similarly close to the image of an ideal politician. The finding can be interpreted with regard to the distinction into sufficient and necessary conditions, where a necessary condition is one which must be present in order for the event to occur but it does not guarantee the event, while a sufficient condition is a condition that will produce the event. Thus, it seems that the list of necessary conditions to be deemed as inadequate for the post is much shorter than the one for an ideal politician.

Overall, our research provides valuable insight into positive-negative asymmetry with regard to a lessexplored area of a differentiation between positive and negative options. Contrary to the findings on the negativity effect and density hypothesis suggesting higher discrepancies between negative options, we find evidence for the opposite effect, showing that people actually see greater differences between a few favourable objects than the negative ones. As our studies were conducted in a setting of political elections, we added valuable insight into the perception of difference among differently valenced social objects - a topic that has been so far less investigated.

Still, our research is not free from limitations. First, taking into consideration no effect of additional positive features when provided in a narrative form (Study 3), further studies should analyse in more detail the differences in the perception of change for stimuli presented in a narrative, numeric and pictorial form. Furthermore, the use of a rather simple form of candidate presentation may pose certain limitations on the ecological validity of the study. Although, the use of such profiles was justified by our intention to have a maximal control over analysed stimuli, further studies should investigate more complex stimuli. These stimuli do not have to be political as our predictions are general in nature. Still, scholars in the field of political branding may consider the moderating role of party affiliation in the differentiation between options. For instance, it is interesting to analyse how well people differentiate between candidates that are from their party compared to the members of the opposing party.

\section{References}

1. Bargh, J. A., Chaiken, S., Govender, R. \& Pratto, F. The Generality of the Automatic Attitude Activation Effect. J. Pers. Soc. Psychol. 62, 893-912 (1992).

2. Hoorens, V. Positivity bias. in Encyclopedia of Quality of Life and Well-Being Research. (ed. Michalos, A. C.) 4938-4941 (Springer, 2014).

3. Sears, D. O. The person-positivity bias. J. Pers. Soc. Psychol. 44, 233-250 (1983). doi:10.1037/00223514.44.2.233

4. Willis, J. \& Todorov, A. First impressions: Making up your mind after a 100-ms exposure to a face. Psychol. Sci. 17, 592-598 (2006).

5. Baumeister, R. F., Bratslavsky, E., Finkenauer, C. \& Vohs, K. D. Bad is stronger than good. Rev. Gen. Psychol. 5, 323-370 (2001).

6. Rozin, P. \& Royzman, E. B. Negativity bias, negativity dominance, and contagion. Personal. Soc. Psychol. Rev. 5, 296-320 (2001). 
7. Matlin, M. W. \& Stang, J. D. The Pollyanna Principle. Selectivity in language, memory, and thought. (Schenkman, 1978).

8. Rozin, P., Berman, L. \& Royzman, E. B. Biases in use of positive and negative words across twenty natural languages. Cogn. Emot. 24, 536-548 (2010).

9. Unkelbach, C., Fiedler, K., Bayer, M., Stegmüller, M. \& Danner, D. Why Positive Information Is Processed Faster: The Density Hypothesis. J. Pers. Soc. Psychol. 95, 36-49 (2008).

10. Bernatzi, S. \& Thaler, R. H. Myopic Loss Aversion and the Equity Premium Puzzle. Q. J. Econ. 110, 73-92 (1995).

11. Tversky, A. \& Kahneman, D. Loss Aversion in Riskless Choice: A Reference-Dependent Model. Q. J. Econ. 106, 1039-1061 (1991).

12. Kahneman, D. \& Tversky, A. Prospect Theory: An Analysis of Decision under Risk. Econom. J. Econom. Soc. 47, 263-291 (1979).

13. Brown, J. S. Gradients of approach and avoidance responses and their relation to level of motivation. J. Comp. Physiol. Psychol. 41, 450-465 (1948).

14. Rozin, P., Markwith, M. \& Nemeroff, C. Magical Contagion Beliefs and Fear of AIDS. J. Appl. Soc. Psychol. 22, 1081-1092 (1992).

15. Kahneman, D. \& Tversky, A. Choices, values, and frames. Am. Psychol. 39, 341-350 (1984).

16. Dhar, R. \& Sherman, S. J. The effect of common and unique features in consumer choice. J. Consum. Res. 23, 193-203 (1996).

17. Dhar, R., Nowlis, S. M. \& Sherman, S. J. Comparison Construction Effects on Preference. J. Consum. Res. 26, 293-306 (1999).

18. Anderson, N. H. Averaging versus adding as a stimulus-combination rule in impression formation. J. Exp. Psychol. 70, 394-400 (1965).

19. Anderson, N. H. Cognitive Algebra: Integration Theory Applied to Social Attribution. Adv. Exp. Soc. Psychol. 7, 1-101 (1974).

20. Fiske, S. T. Attention and weight in person perception: The impact of negative and extreme behavior. J. Pers. Soc. Psychol. 38, 889-906 (1980).

21. Bless, H. \& Schwarz, N. Mental construal and the emergence of assimilation and contrast effects: The inclusion/exclusion model. Advances in Experimental Social Psychology 42, (Elsevier Inc., 2010).

22. Schwarz, N. \& Bless, H. Assimilation and Contrast Effects in Attitude Measurement: an Inclusion/Exclusion Model. in NA - Advances in Consumer Research Volume 19 (eds. Sherry Jr., J. F. \& Sterntha, B.) 72-77 (Association for Consumer Research, 1992).

23. Schwarz, N. \& Bless, H. Scandals and the Public's Trust in Politicians: Assimilation and Contrast Effects. Personal. Soc. Psychol. Bull. 18, 574-579 (1992).

24. Gescheider, G. A. Psychophysics: The Fundamentals. (Erlbaum, 1997).

25. Stevens, S. S. On the psychophysical law. Psychol. Rev. 64, 153-181 (1957).

26. Tversky, A. Features of Similarity. Psychol. Rev. 84, 327-352 (1977). 
27. Falkowski, A., Sidoruk-Błach, M., Olszewska, J. \& Jabłońska, M. Positive-negative asymmetry in evaluation of natural stimuli: Empirical study in the contrast model of similarity extended to open sets. Am. J. Psychol. 134, 1-11 (2021).

28. Falkowski, A. \& Jabłońska, M. Positive-Negative Asymmetry in the Evaluations of Political Candidates. The Role of Features of Similarity and Affect in Voter Behavior. Front. Psychol. 9, 213 (2018).

29. APA. Ethical Principles of Psychologists and Code of Conduct. (2010). Available at: http://www.apa.org/ethics/code/. (Accessed: 3rd March 2016)

30. Bizer, G. Y. \& Petty, R. E. How We Conceptualize Our Attitudes on the Resistance The Effects of Valence Framing of Political Attitudes. Polit. Psychol. 26, 553-568 (2012).

31. Cwalina, W., Falkowski, A. \& Kaid, L. L. Advertising and the image of politicians in evolving and established democracies: Comparative study of the Polish and the US presidential elections in 2000. J. Polit. Mark. 4, 19-44 (2005).

32. Cwalina, W. \& Falkowski, A. Political Communication and Advertising in Poland. in The Sage Handbook of Political Advertising (eds. Kaid, L. L. \& Holtz-Bacha, C.) 325-466 (SAGE Publications, 2006).

33. Kinder, D. R., Peters, M. D., Abelson, R. P. \& Fiske, S. T. Presidential prototypes. Polit. Behav. 2, 315337 (1980).

34. Wessa, P. Skewness and Kurtosis Test (v1.0.4). Free Statistics Software (v1.2.1). Office for Research Development and Education (2017).

35. Bewick, V., Cheek, L. \& Ball, J. Statistics review 10: Nonparametric methods. Crit. Care 8, 196 (2004).

36. Yu, C. H. Resampling methods: Concepts, applications, and justification. Pract. Assessment, Res. Eval. 8, Article 19 (2002).

37. Welch, W. J. Construction of permutation tests. J. Am. Stat. Assoc. 85, 693-698 (1990).

38. Team, Rs. RStudio: Integrated Development for R. RStudio, Inc. (2015).

39. Cohen, J. Statistical power analysis for the behavioral sciences. Statistical Power Analysis for the Behavioral Sciences (1988). doi:10.1234/12345678

40. Kirkpatrick, L. A. \& Epstein, S. Cognitive-experiential self-theory and subjective probability: further evidence for two conceptual systems. J. Pers. Soc. Psychol. 63, 534-544 (1992).

41. Epstein, S. Cognitive experiential self theory. in Handbook of Personality: Theory and Research (ed. Pervin, L.) 165-192 (Guilford Press, 1990).

\section{Figures}




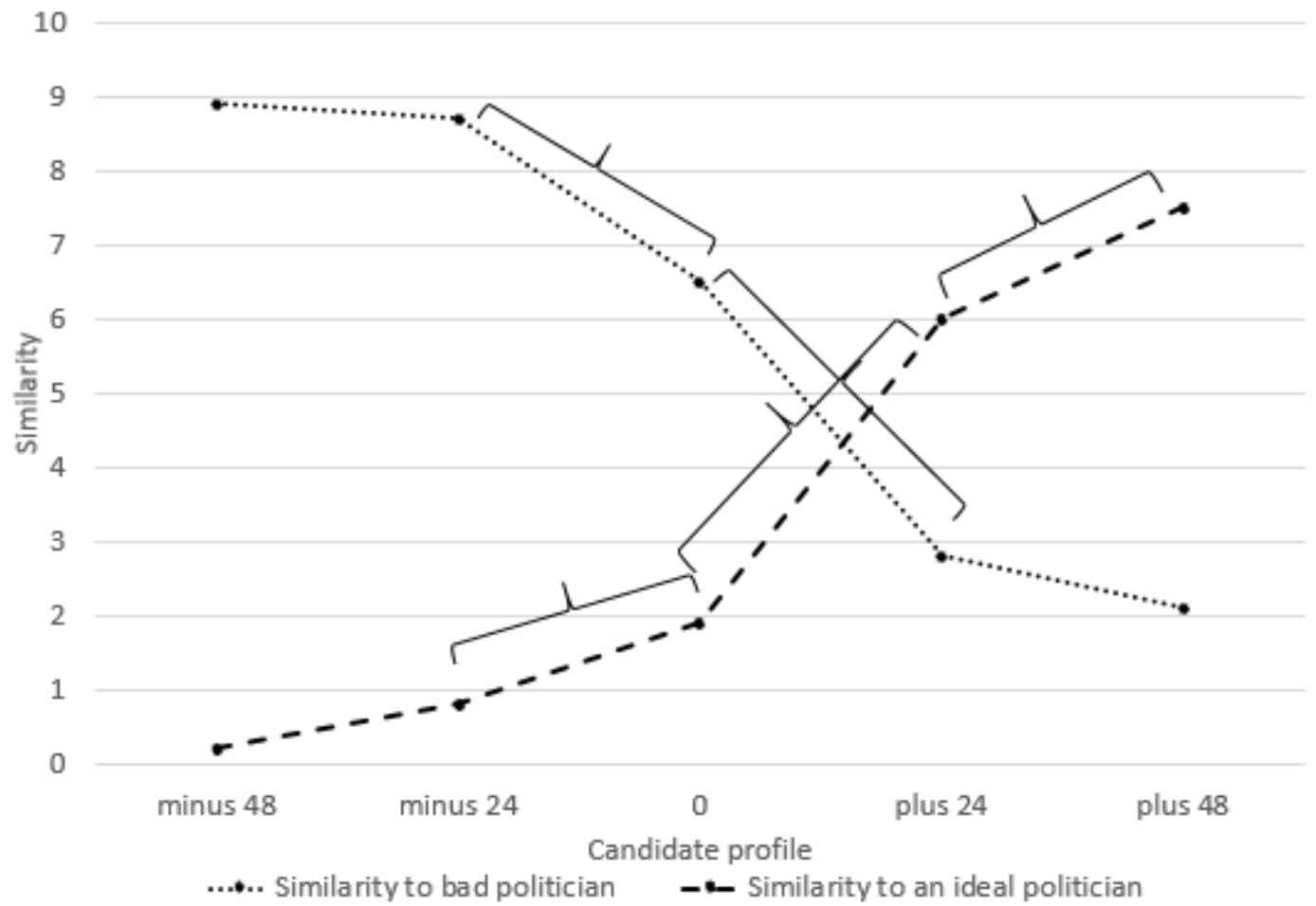

\section{Figure 1}

Similarity to an ideal and bad politician for candidates analysed in Study 1 . Captions minus 48, minus 24, 0 , plus 24 and plus 48 refer to the overall valence of candidate profiles. Brackets mark significant differences $(p<.01)$ between the analysed candidates.

\section{Supplementary Files}

This is a list of supplementary files associated with this preprint. Click to download.

- Appendix1.docx 